\title{
ANÁLISE DA QUALIDADE DA ÁGUA NA BACIA HIDROGRÁFICA DO RIO IGARAPÉ DA FORTALEZA - AMAPÁ
}

\author{
L. de A. da SILVA ${ }^{1}$, L. R. de $\operatorname{SOUZA}^{2}$ e A. C. da CUNHA ${ }^{3}$ \\ ${ }^{1}$ Universidade do Estado do Amapá, Departamento de Engenharia Química \\ ${ }^{2}$ Universidade Federal do Amapá, Departamento de Física \\ ${ }^{3}$ Universidade Federal do Amapá, Departamento de Ciências Ambientais \\ Email para contato: lurdy1874@ hotmail.com
}

\begin{abstract}
RESUMO - Há poucos estudos na literatura sobre a qualidade da água em bacias hidrográficas sob influência de marés nas várzeas da Amazônia, especialmente como suporte de avaliação de impactos de poluição orgânica em cursos naturais de água. O objetivo deste trabalho foi identificar possíveis poluições em um trecho de $6 \mathrm{~km}$ de extensão do rio urbano Igarapé da Fortaleza$\mathrm{AP}$, típico da várzea, através de estudos experimentais de parâmetros como demanda bioquímica de oxigênio (DBO), nitrogênio amoniacal e oxigênio dissolvido (OD). A metodologia consistiu em coletas de amostras da água do rio durante o ciclo de maré semi-diurno (12,5h), seguida de uma análise laboratorial. Como resultado, foram obtidos concentrações de condutividade, ferro, fósforo e oxigênio dissolvido fora do padrão segundo a resolução 357 do Conselho regional de Meio Ambiente (CONAMA). Logo, o monitoramento contínuo no rio faz-se necessário para criar métodos que minimizem a poluição, garantindo assim sua qualidade e conservação.
\end{abstract}

\section{INTRODUÇÃO}

Os corpos hídricos da região costeira de Macapá e Santana tem sido severamente alterados em sua qualidade. Por outro lado, tais efeitos deletérios são também compensados pelos processos de autodepuração natural do rio, devido principalmente aos efeitos das marés semidiurnas destes ambientes (CUNHA, 2004).

O fenômeno da poluição localizada e difusa próximas de centros urbanos, como é o caso do Igarapé da Fortaleza (IF), está se expandindo para diversos municípios do Estado do Amapá. Portanto, não está restrito apenas à "poluição" devido as interferências advindas das atividades antrópicas domésticas, mas também da construção de hidrelétricas, inserção de indústrias, expansão da agrigultura e pecuária, além de outros usos e ocupação desordenados do solo nas bacias hidrográficas (BASTOS, 2010; BÁRBARA, 2006; CUNHA e COUTO, 2002).

Para coibir tais efeitos crescentes e intensivos nas bacias hidrográficas dispõe-se de regulamentação dos padrões da qualidade da água dos rios no Brasil. Uma das mais citadas é a Resolução 357 de 17 de março de 2005 do CONAMA, a qual dispõe sobre a classificação 
dos corpos de água e diretrizes ambientais, estabelecendo condições e padrões de lançamento de efluentes (BRITO, 2008). Para o gerenciamento dos recursos hídricos, também foi criada no Amapá a lei 0686 de 7 de junho 2002 (AMAPÁ, 2002), porém esta necessita de regulamentação, devido à falta de estudos para subsidiá-los no Estado.

O estado do Amapá, com aproximadamente $143.000 \mathrm{Km}^{2}$ de superfície, possui o terceiro maior volume de descarga de rios do Brasil (REBOUÇAS, 2004). Possui 33 bacias hidrográficas (Lei 0686, 2002) e está inserido totalmente na bacia amazônica, onde encontramos o maior volume de água doce do mundo. A bacia do IF está localizada na zona da costa estuarina do Estado do Amapá, cujo curso principal deságua no Rio Amazonas (CUNHA, 2004).

Com o objetivo de dar suporte às instituições de fiscalização e controle da qualidade das águas, o presente estudo contribui como mais um suporte às estratégicas políticas de gerenciamento e conservação da referida Bacia Hidrográfica e aos recursos hídricos nestes ecossistemas desconhecidos como afirmam BASTOS (2010) e CUNHA e COUTO (2002). Deste modo foram analisados experimentalmente seis (6) parâmetros físico-químicos da água na Bacia Hidrográfica do Rio Igarapé da Fortaleza (BHIF), e avaliado seu comportamento espaço-temporal (semidiurno) em dois períodos sazonais (chuvoso e seco) observando-se sua real qualidade considerando-se os processos autodepurativos envolvidos próximos de sua foz.

\section{MATERIAL E MÉTODOS}

\section{1 Área em estudo - Bacia Hidrográfica do Rio Igarapé da Fortaleza}

O Estado do Amapá, localizado no extremo norte do Brasil, tem uma rede hídrica bastante significativa dentro dos domínios da Amazônia. Completamente dentro dos seus limites está a Bacia Hidrográfica do Rio Igarapé da Fortaleza.

A pesquisa foi realizada em um trecho da BHIF, compreendido entre a Foz e até cerca de $6 \mathrm{~km}$ a montante, no qual está situado entre os municípios de Macapá (AP) e Santana (AP), conforme mostra a Figura 1a (Bacia Hidrográfica), b e c (sítios de coleta 1, 2 e 3): 


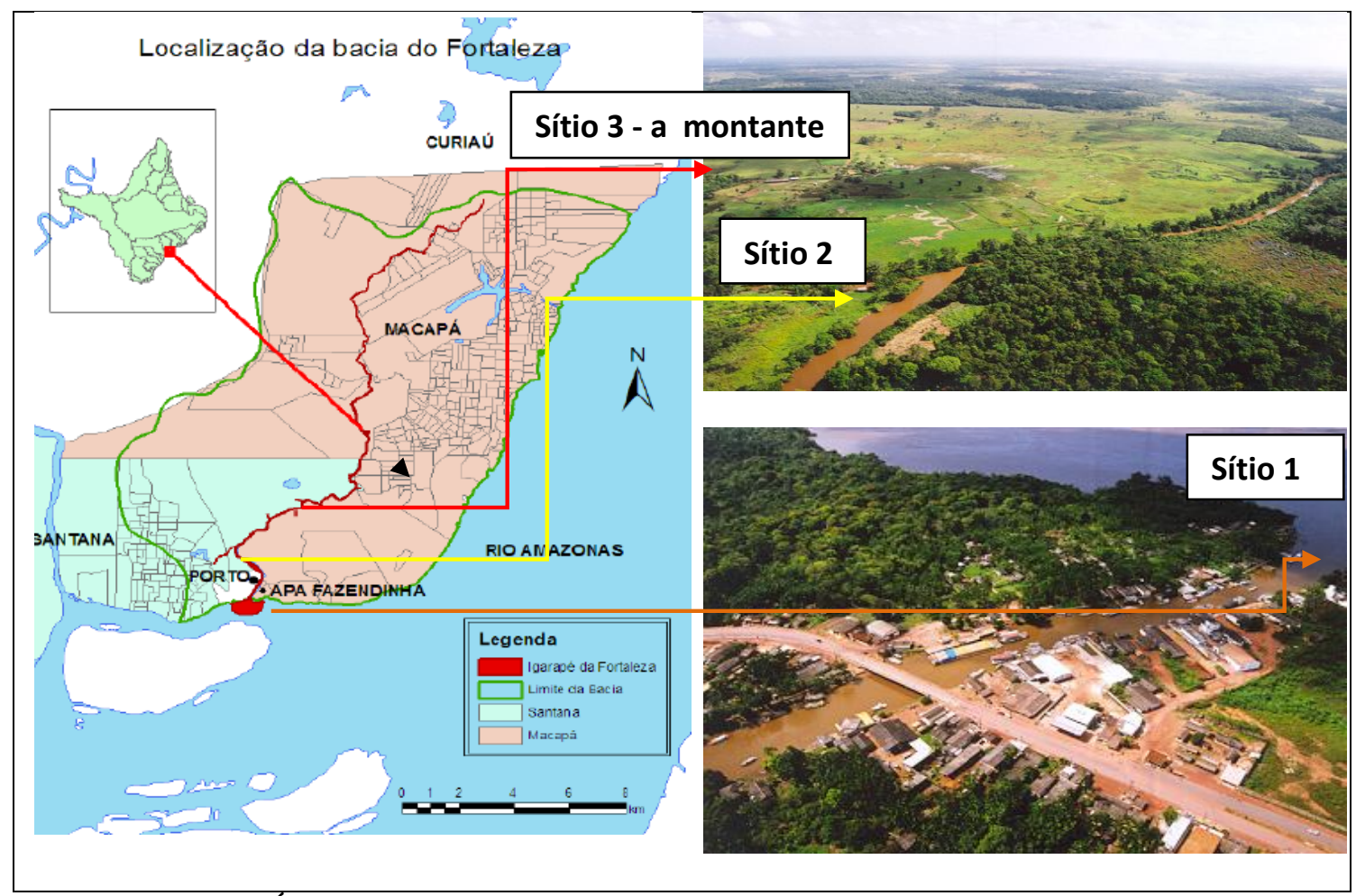

Figura 1: Área em estudo situando o igarapé entre os municípios de Macapá e Santana.

Fonte: BASTOS (2010, CUNHA e COUTO, 2002)

O Igarapé da Fortaleza (IF) apresenta extensão de 27,3 Km com sua nascente no bairro Sol Nascente localizado na zona norte de Macapá atravessando área de ressaca e florestas de várzea desemborcando no rio Amazonas (BASTOS, 2010). Mas na presente pesquisa foi estudado seu trecho inferior, com aproximadamente $6 \mathrm{~km}$ de extensão (Figura 1), com frequência amostral a aproximadamente cada $4 \mathrm{~h}$.

\subsection{Monitoramento da qualidade da água}

\section{Procedimento experimental em campo}

As coletas foram realizadas em intervalos próximos de $4 \mathrm{~h}$, por cerca de $12,5 \mathrm{~h}$ seguidas, abrangendo o tempo de preia-mar e baixa-mar. Sendo que a qualidade da água foi monitorada em 3 pontos, descritos na Tabela 1.

Tabela 1: Pontos nos quais as coletas foram realizadas.

\begin{tabular}{c|l|l}
\hline \multicolumn{1}{c|}{ Sítio 1 } & \multicolumn{1}{|c|}{ Sítio 2 } & \multicolumn{1}{c}{ Sítio 3 } \\
\hline $\begin{array}{l}\text { Foz da Bacia Hidrográfica } \\
\text { Igarapé da Fortaleza }\end{array}$ & $\begin{array}{l}\text { Antes da entrada do } \\
\text { Igarapé do Provedor }\end{array}$ & $\begin{array}{l}\text { Cerca de 6 km a montante } \\
\text { do Igarapé da Fortaleza. }\end{array}$ \\
\hline
\end{tabular}

Em campo, foram obtidos dados imediatos dos parâmetros de condutividade e sólidos totais dissolvidos, obtidos através da sonda (HACH COMPANY, 2005). Além disso, ainda em campo foram coletadas amostras em frascos poliméricos e de $\mathrm{BDO}_{(5,20)}$, para uma conseguinte análise laboratorial dos parâmetros de ferro, fósforo, nitrogênio amoniacal, oxigênio dissolvido e demanda bioquímica de oxigênio. 


\section{$\underline{\text { Procedimento experimental em laboratório }}$}

A escolha dos métodos, materiais e reagentes necessários para a coleta, transporte, conservação e análises das amostras, bem como para selecionar os equipamentos que foram transportados para os locais de amostragem, foram consultados em manuais e materiais bibliográficos adaptados do Standard Methods for theExamination of Water and Wastewater (HACH COMPANY, 2005).

\section{RESULTADOS E DISCUSSÃO}

Os resultados das análises experimentais dos parâmetros analisados são mostrados na Tabela 3, e comparados com os valores da resolução 370/2005 e 274/2000 do Conselho Nacional de Meio Ambiente (CONAMA), os quais foram verificados sua conformidade com os padrões aceitáveis pela referida norma. Os valores máximos padrões de alguns parâmetros classe II analisados para esta categoria, são de águas que podem ser destinadas ao abastecimento e consumo humano, após tratamento convencional; à proteção das comunidades aquáticas; à recreação de contato primário, tais como natação, esqui aquático e mergulho, conforme Resolução CONAMA no 274, de 2000; à irrigação de hortaliças, plantas frutíferas e de parques, jardins, campos de esporte e lazer, com os quais o público possa vir a ter contato direto e à aqüicultura e à atividade de pesca, disponíveis na Tabela 2. Observa-se que todos estes usos da água ocorrem com frequência (CUNHA e COUTO, 2002; CUNHA, 2004; BASTOS, 2010)

Tabela 2. Valores máximos de aceitação para águas doces, de classe 2

\begin{tabular}{l|l}
\hline \multicolumn{1}{c|}{ Parâmetros } & \multicolumn{1}{c}{ Valor Máximo } \\
\hline DBO $_{(5,20)}$ & Até $5 \mathrm{mg} / \mathrm{L} \mathrm{O} \mathrm{O}_{2}$ \\
\hline Ferro & $0,3 \mathrm{mg} / \mathrm{L}$ \\
\hline Fósforo & $0,1 \mathrm{mg} / \mathrm{L}$ \\
\hline & $3,7 \mathrm{mg} / \mathrm{L} \mathrm{N}$, para $\mathrm{pH} \leq 7,5$ \\
Nitrogênio amoniacal total & $2,0 \mathrm{mg} / \mathrm{L} \mathrm{N}$, para $7,5<\mathrm{pH} \leq 8,0$ \\
& $1,0 \mathrm{mg} / \mathrm{L} \mathrm{N}$, para $8,0<\mathrm{pH} \leq 8,5$ \\
& $0,5 \mathrm{mg} / \mathrm{L} \mathrm{N}$, para $\mathrm{pH}>8,5$ \\
\hline Oxigênio Dissolvido & Não inferior a $5 \mathrm{mg} / \mathrm{L}$ \\
\hline
\end{tabular}


Tabela 3: Dados experimentais das coletas realizadas no Igarapé da Fortaleza, durante o período chuvoso e seco.

\begin{tabular}{|c|c|c|c|c|c|c|c|c|c|c|c|c|}
\hline \multicolumn{13}{|c|}{ Coleta realizada no período chuvoso $(13 / 06 / 2013)$} \\
\hline \multirow[b]{2}{*}{ Parâmetros } & \multicolumn{3}{|c|}{ Primeira coleta } & \multicolumn{3}{|c|}{ Segunda coleta } & \multicolumn{3}{|c|}{ Terceira coleta } & \multicolumn{3}{|c|}{ Quarta coleta } \\
\hline & $\begin{array}{c}\text { Ponto } \\
1\end{array}$ & $\begin{array}{c}\text { Ponto } \\
2\end{array}$ & $\begin{array}{c}\text { Ponto } \\
3\end{array}$ & $\begin{array}{c}\text { Ponto } \\
1\end{array}$ & $\begin{array}{c}\text { Pont } \\
\text { o } 2\end{array}$ & $\begin{array}{c}\text { Ponto } \\
3\end{array}$ & $\begin{array}{c}\text { Ponto } \\
1\end{array}$ & $\begin{array}{c}\text { Ponto } \\
2\end{array}$ & $\begin{array}{c}\text { Ponto } \\
3\end{array}$ & $\begin{array}{c}\text { Ponto } \\
1\end{array}$ & $\begin{array}{c}\text { Ponto } \\
2\end{array}$ & $\begin{array}{c}\text { Ponto } \\
3\end{array}$ \\
\hline Condutividade $(\mu \mathrm{s} / \mathrm{cm})$ & 46 & 47 & 63 & 50 & 61 & 64 & 63 & 72 & 62 & 46 & 63 & 66 \\
\hline Ferro $(\mathrm{mg} / \mathrm{L} \mathrm{Fe})$ & 1,12 & 1,15 & 1,32 & - & 1,46 & 1,18 & 1,32 & 1,27 & 1,16 & 1,16 & 1,44 & 1,36 \\
\hline Fósforo $\left(\mathrm{mg} / \mathrm{L} \mathrm{PO}_{4}^{-3}\right)$ & 0,36 & 0,50 & 0,47 & 0,34 & 0,47 & 0,58 & 0,46 & 0,85 & 0,61 & 0,24 & 0,76 & 0,99 \\
\hline Nitrogênio amoniacal total $(\mathrm{mg} / \mathrm{L}) \mathrm{NH}_{3}-\mathrm{N}$ & 0,52 & 0,76 & 0,66 & 0,59 & 0,56 & 0,74 & 0,68 & 0,67 & 0,78 & 0,54 & 0,74 & 0,99 \\
\hline Oxigênio Dissolvido (mg/L) & 4,14 & 4,25 & 2,90 & 4,07 & 3.52 & 2,35 & 3,21 & 2,92 & 2,91 & 4,56 & 3,07 & 2,66 \\
\hline Sólidos totais dissolvidos $(\mathrm{mg} / \mathrm{L})$ & 300 & 300 & 400 & 320 & 390 & 420 & 410 & 470 & 400 & 300 & 420 & 430 \\
\hline \multicolumn{13}{|c|}{ Coleta realizada no período seco $(20 / 10 / 2013)$} \\
\hline & \multicolumn{3}{|c|}{ Primeira coleta } & \multicolumn{3}{|c|}{ Segunda coleta } & \multicolumn{3}{|c|}{ Terceira coleta } & \multicolumn{3}{|c|}{ Quarta coleta } \\
\hline Parâmetros & $\begin{array}{c}\text { Ponto } \\
1\end{array}$ & $\begin{array}{c}\text { Ponto } \\
2\end{array}$ & $\begin{array}{c}\text { Ponto } \\
3\end{array}$ & $\begin{array}{c}\text { Ponto } \\
1\end{array}$ & $\begin{array}{c}\text { Ponto } \\
2\end{array}$ & \begin{tabular}{|c} 
Ponto \\
3
\end{tabular} & \begin{tabular}{|c} 
Ponto \\
1
\end{tabular} & $\begin{array}{c}\text { Ponto } \\
2\end{array}$ & $\begin{array}{c}\text { Ponto } \\
3\end{array}$ & $\begin{array}{c}\text { Ponto } \\
1\end{array}$ & $\begin{array}{c}\text { Ponto } \\
2\end{array}$ & $\begin{array}{c}\text { Ponto } \\
3\end{array}$ \\
\hline Condutividade $(\mu \mathrm{s} / \mathrm{cm})$ & 52 & 52 & 66 & 53 & 55 & 84 & 54 & 70 & 139 & 55 & 83 & 172 \\
\hline $\mathrm{DBO}_{(5,20)}(\mathrm{mg} / \mathrm{L})$ & 0,83 & 0,69 & 0,67 & 0,41 & 0,45 & 0,80 & 0,98 & 1,32 & 1,34 & 0,46 & 1,41 & 1,18 \\
\hline Ferro $(\mathrm{mg} / \mathrm{L} \mathrm{Fe})$ & 0,9 & 1,08 & 1,35 & 0,92 & 1,19 & 1,41 & 1,11 & 1,41 & 1,37 & 0,88 & 1,39 & 1,31 \\
\hline Fósforo $\left(\mathrm{mg} / \mathrm{L} \mathrm{PO}_{4}^{-3}\right)$ & 0,82 & 0,51 & 0,56 & 0,61 & 0,63 & 0,51 & 0,55 & 0,54 & 0,65 & 0,67 & 0,82 & 0,83 \\
\hline Nitrogênio amoniacal total $(\mathrm{mg} / \mathrm{L}) \mathrm{NH}_{3}-\mathrm{N}$ & 0,60 & 0,61 & 0,59 & 0,61 & 0,65 & 0,54 & 0,58 & 0,58 & 0,47 & 0,63 & 0,55 & 0,71 \\
\hline Oxigênio Dissolvido (mg/L) & 6,63 & 6,33 & 4,87 & 5,98 & 5,66 & 4,34 & 5,98 & 5,29 & 4,05 & 6,37 & 4,61 & 2,79 \\
\hline Sólidos totais dissolvidos (mg/L) & 330 & 340 & 430 & 340 & 360 & 550 & 350 & 450 & 910 & 350 & 540 & 1120 \\
\hline
\end{tabular}


Em analogia com os valores provenientes de campo e os valores padrões fornecidos pela Resolução CONAMA (357/05) foi possível realizar a seguinte análise:

\section{$\underline{\text { Análise da condutividade elétrica }}$}

Os valores de condutividade encontrados nos pontos amostrais variaram entre $46 \mu \mathrm{s} / \mathrm{cm}$ a $72 \mu \mathrm{s} / \mathrm{cm}$ no inverno e $52 \mu \mathrm{s} / \mathrm{cm}$ a $172 \mu \mathrm{s} / \mathrm{cm}$ no período seco. Estes valores são diretamente proporcionais a concentração de sólidos totais dissolvidos, ocasionados principalmente pela produção de íons presentes no esgoto, ou de erosão de solos sedimentares da bacia a montante (CUNHA e COUTO, 2002), e derramamento de combustíveis próximo da zona portuária do IF.

$\mathrm{Na}$ referida Resolução não há referência a um valor limite deste indicador. Mas, segundo CETESB (2013) somente limites superiores a $100 \mu \mathrm{Scm}^{-1}$ indicam ambientes impactados. Logo, os valores relacionados a este parâmetro ainda estavam satisfatórios em comparação às amostras em geral coletadas, mas com dois valores obtidos fora do padrão (139 e $172 \mu \mathrm{S} / \mathrm{cm}$ ) durante o período seco (Tabela 3, sítio amostral 3).

\section{$\underline{\text { Análise de Demanda Bioquímica de Oxigênio }\left(\mathrm{DBO}_{(5,20)}\right)}$}

Infelizmente este parâmetro não pôde ser medido na coleta realizada no período chuvoso, devido problemas de equipamento para a sua realização.

$\mathrm{A} \mathrm{DBO}_{(5,20)}$ é um dos principais indicadores de poluição nas águas de um rio (matéria orgânica respirável), e os valores obtidos deste parâmetro na BHIF mostraram-se satisfatórios, haja vista que suas medidas variaram desde 0,41 até $1,41 \mathrm{mgL}^{-1}$, valores bem abaixo do estipulado pela resolução 357/05 do CONAMA, próximos de 5mg/L (Tabela 2).

\section{Análise de ferro dissolvido $\left(\mathrm{Fe}^{+2}\right)$}

A resolução 357/05 do CONAMA estipula como padrão máximo a concentração de $0,3 \mathrm{mgL}^{-1}$ de ferro dissolvido nas águas de rio. Assim, a BHIF contribui com significativa elevação nos níveis de ferro em todas as amostras analisadas, visto que as variações de 1,12 $\mathrm{mg} / \mathrm{L} \mathrm{Fe}$ a $1,46 \mathrm{mg} / \mathrm{L} \mathrm{Fe}^{+2}$ no período chuvoso foi de $0,88 \mathrm{mg} / \mathrm{L} \mathrm{Fe} \mathrm{a} 1,41 \mathrm{mg} / \mathrm{L} \mathrm{Fe}$ no seco. Essas concentrações podem ser justificadas principalmente pelo fato de que os rios refletem as características dos solos regionais, ricos em $\mathrm{Fe}$, mas também uma parcela dessas concentrações pode ser de origem de esgoto doméstico (BRITO, 2008; BÁRBARA, 2010 e REBOUÇAS, 2004).

\section{$\underline{\text { Análise de fósforo total }(\mathrm{P})}$}

A medida deste parâmetro é fundamental para subsidiar estudos em ecologia, principalmente sua influência sobre as algas na região, pois o fósforo é um importante nutriente para os processos biológicos e seu excesso pode causar a eutrofização das águas (REBOUÇAS, 2004).

Os valores de fósforo total, encontrados no trecho em estudo, variaram entre 0,24 $\mathrm{mg} / \mathrm{L}$ a $0,99 \mathrm{mg} / \mathrm{L}$ no período chuvoso e $0,51 \mathrm{mg} / \mathrm{L}$ a $0,83 \mathrm{mg} / \mathrm{L}$ no seco, valores muito acima do padrão estabelecido pela resolução 357/05 do CONAMA, a qual estipula para ambientes lóticos (ambiente relativo a águas continentais dinâmicas), como é o caso do BHIF, o valor foi de $0,1 \mathrm{mgL}^{-1}$. 
Infere-se que estas altas concentrações de fósforo estejam relacionadas principalmente por esgotos domésticos, pela presença dos detergentes superfosfatados e da própria matéria orgânica fecal, problema encontrado no trecho em estudo, em função da ocupação urbana desordenada em torno do rio (CUNHA, 2004).

\section{$\underline{\text { Análise de nitrogênio amoniacal }\left(\mathrm{NH}_{3}-\mathrm{N}\right)}$}

O nitrogênio amoniacal é formado por processos de decomposição de matéria orgânica, que sofre decomposição até o íon nitrato, passando inicialmente pela amônia. Este parâmetro normalmente é um indicador de poluição recente. Contudo, os níveis de concentração deste parâmetro no rio foram satisfatórios e dentro dos padrões estabelecidos pela resolução 357/05 do CONAMA, a qual estipula $3,7 \mathrm{mgL}^{-1}$ como limite máximo, para $\mathrm{pH}$ menor ou igual a 7,5, como é o caso da água da bacia $(\mathrm{pH}<6,5-\mathrm{CUNHA}, 2004)$. Sua concentração máxima foi de $0,99 \mathrm{mgL}^{-1}$ e mínima de $0,52 \mathrm{mgL}^{-1}$ no período chuvoso e respectivamente $0,71 \mathrm{mgL}^{-1}$ e $0,47 \mathrm{mgL}^{-1}$ no seco, tendo como provável fonte esgotos sanitários.

\section{Análise de oxigênio dissolvido}

Como estabelecido pela resolução do CONAMA 357/05 para rios de classe 2, os teores de oxigênio devem ser no mínimo de $5 \mathrm{mg} . \mathrm{L}^{-1} \mathrm{O}_{2}$. De acordo com as análises realizadas, o ambiente em estudo não se mostrou bem oxigenado durante o período chuvoso, pois as concentrações de oxigênio dissolvido (OD) tiveram uma variação de $2,35 \mathrm{mg} . \mathrm{L}^{-1} \mathrm{O}_{2}$ a 4,56 mg. $\mathrm{L}^{-1} \mathrm{O}_{2}$. Enquanto que no período seco os valores variaram entre $2,71 \mathrm{mg} . \mathrm{L}^{-1} \mathrm{O}_{2}$ a $6,63 \mathrm{mg} . \mathrm{L}^{-1} \mathrm{O}_{2}$, obtendo-se assim, uma melhora parcial de oxigenação.

Uma análise mais cuidadosa deste parâmetro sugere que as águas poluídas por esgotos (poluição difusa principalmente) apresentam baixa concentração de oxigênio dissolvido, devido a carga orgânica consumida no processo de decomposição. Assim, esta pode ser uma das principais causas da baixa concentração de oxigênio na BHIF, como afirma BASTOS (2010).

\section{Análise de sólidos totais dissolvidos}

Os valores deste parâmetro variaram entre $300 \mathrm{ppm}$ a $470 \mathrm{ppm}$ no período chuvoso e 330 ppm a 1120 ppm no seco. Assim, as concentrações da coleta do período chuvoso estão todas em conformidade com a legislação. Porém, na coleta do período seco, alguns dados do ponto $3(25 \%)$ se mostraram alarmantes, pois neste ponto foram obtidas concentrações bem superiores ao padrão. Estes problemas ambientais podem ser reflexos causados pelo uso e ocupação devido ao desmatamento, erosão e assoreamento, conforme pode ser observado pela Figura 1 b e c.

\section{CONCLUSÃO}

A partir da análise realizada entre os dados experimentais e valores padrões disponíveis nas resoluções 287 e 357 do Conselho Regional de Meio Ambiente (CONAMA), foi possível verificar concentrações de parâmetros como condutividade, ferro, fósforo, oxigênio dissolvido e sólidos totais dissolvidos fora do padrão. Portanto, o monitoramento contínuo na área em estudo faz-se necessário para se dispor de informações 
confiáveis da qualidade da água, visando posteriormente o monitoramento sistemático para buscar soluções de mitigação da poluição.

\section{REFERÊNCIA BIBLIOGRÁFICA}

BÁRBARA, V. F. Uso do Modelo QUAL2E no estudo da qualidade da água e da capacidade de autodepuração do Rio Araguari - AP (Amazônia). 2006. 174 p. Dissertação (Mestrado em Engenharia do Meio Ambiente), Universidade Federal de Goiás, GoiâniaGoiás, 2006.

BASTOS, A. M. Modelagem de escoamento ambiental como subsídio à gestão de ecossistemas aquáticos no baixo Igarapé da Fortaleza. 2010. 117 p. Dissertação (Mestrado em Biodiversidade Tropical), Universidade Federal do Amapá. Macapá-Amapá. 2010.

BRITO, D. C. Aplicação do sistema de modelagem da qualidade da água QUAL2Kw em grandes rios: o caso do alto e médio rio Araguari-AP. 2008.152 p. Dissertação (Mestrado em Biodiversidade Tropical), Universidade Federal do Amapá. Macapá-Amapá. 2008.

CETESB - COMPANHIA DE TECNOLOGIA DE SANEAMENTO AMBIENTAL. Qualidade das água interiores no estado de São Paulo. São Paulo. Disponível em: < http://www.cetesb.sp.gov.br/userfiles/file/agua/aguas-superficiais/variaveis.pdf $>$. Acesso em: 16 de agosto de 2013.

CONAMA - CONSELHO NACIONAL DO MEIO AMBIENTE. Resolução n 357, de 18 de junho de 2005. Disponível em: <http://www.mma.gov.br/port/conama/ /res35705.pdf>. Acesso em: 25 de jun de 2013.

CUNHA, A. C. Qualidade Microbiológica da Água em Rios de Áreas Urbanas e Periurbanas no Baixo Amazonas: O Caso do Amapá. Engenharia Sanitária Ambiental, v. 9, n. 4, p. 322328, 2004.

CUNHA, A. C. e COUTO, Diagnóstico Rápido Participativo (DRP) do Igarapé da Fortaleza Secretaria de Estado de Ciência e Tecnologia do Amapá (SETEC-AP). 54 p. 2002.

HACH COMPANY. DR/2800 ESPECTROFOTOMETER. Procedures Manual. $1^{\mathrm{a}}$ ed. Germany.2005.

LEI 0686, de 07 de junho de 2002. In: Políticas de Gerenciamento dos Recursos Hídricos do Estado do Amapá. Macapá, AP, GEA/SEMA, p.59, 2002.

REBOUÇAS, A. C. Uso Inteligente da Água. São Paulo, SP: Escrituras, 2004. 207 p. 